\title{
Monika Braß: \\ Der Zettelkasten hat ausgedient - Digitalisierung der Alphabetischen Kataloge an der WLB
}

Im neuen Jahr werden den Benutzern der Landesbibliothek der alphabetische Hauptkatalog sowie die alphabetischen Kataloge der Bibliothek für Zeitgeschichte und der Musikabteilung online als digitalisierte Kartenkataloge zur Verfügung stehen. Damit ist das Ende des Zeitalters der Zettelkataloge eingeläutet, wenngleich ihr Ordnungsprinzip auch im Imagekatalog noch fortleben wird. Die Preußischen Instruktionen haben schon mehrere Katalogbrüche in unserem Haus überstanden und nun auch Eingang in die Welt der Online-Kataloge gefunden. Dass sie durch die geplante Volltextsuche in den digitalisierten Kartenimages weiter an Bedeutung verlieren werden, kann als sicher gelten, ob sie durch eine vollständige Konversion des Zettelkataloges jemals ganz der Geschichte angehören werden, ist angesichts der angespannten Haushaltslage mehr als fraglich.

Der Anfang vom Ende des konventionellen Zettelkataloges jedenfalls ist da, genauer gesagt begann er schon vor einiger Zeit, nämlich Ende der 80er Jahre, als die Verbundkatalogisierung aufgenommen wurde und der Zettelkatalog nur noch mit Nachweisen von Literatur bis 1988 bestückt wurde. Dennoch behielt er als einziges vollständiges Nachweisinstrument der Be- stände bis 1988 eine zentrale Funktion für die alphabetische Suche. Es schien daher naheliegend, auch diesen Katalog für zeit- und ortsunabhängige Online-Recherchen im Internet zugänglich zu machen. Eine jüngst durchgeführte Benutzerumfrage bestätigt diese Einschätzung. Der konventionelle Weg zu diesem Ziel, die Konversion der PITitelaufnahmen, wäre viel zu zeit- und kostenintensiv gewesen.

Die Entdeckung einer Asbestbelastung und die dadurch beschleunigte Umgestaltung der Leihstelle besiegelten schließlich das Schicksal des Zettelkataloges: Er sollte dem geplanten Selbstabholungsbereich weichen, seine Digitalisierung und Erschließung durch einen alphabetischen Index wurde beschlossen, eine Volltextrecherche als zweite Stufe der Erschließung avisiert.

\section{Projektverlauf}

Zu Beginn dieses Jahres nahm das Projekt konkrete Gestalt an. Eine Arbeitsgruppe wurde gebildet, die nach einer kurzen Phase des Erfahrungsaustausches mit anderen Bibliotheken daran ging, eine Leistungsbeschreibung für das Projekt zu entwerfen, die als Kernstück einer späteren Ausschreibung dienen sollte. 
Im Zentrum dieses Pflichtenheftes stand die Forderung, dass die Katalogkarten vor Ort in der Bibliothek gescannt werden, und zwar bitonal mit einer Auflösung von mindestens 300 dpi und der Option, die Daten später mittels Texterkennung für eine Volltextrecherche aufzubereiten. Um eine komfortable Navigation im Katalog zu ermöglichen, wurde festgelegt, dass bei der Indexierung jede 50 . Karte sowie die bereits im Zettelkatalog befindlichen Leitkarten zu berücksichtigen seien. Die Aufbereitung der Daten für die Online-Recherche sowie die Recherche-Software sollte von der hauseigenen EDV-Abteilung entwickelt werden. Als Projektschluss wurde der 31. Oktober 2003 festgelegt. Von den fünf Firmen, die sich am Ausschreibungsverfahren beteiligten, erhielt die Firma Mikro Univers aus Berlin am 5.
Juni den Zuschlag.

Das Imagekatalog-Team ging nun daran, eine detaillierte Anleitung für die Indexierung der Katalogkarten zu erstellen, was sich angesichts der Besonderheiten des Pl-Regelwerkes als keine ganz triviale Aufgabe erwies. Grundprinzip der Indexierung ist, dass die im PI-Kopf der Katalogkarten ausgegebenen Ordnungswörter bis einschließlich des ersten Ordnungswortes, das sich vom vorhergehenden und vom nachfolgenden Eintrag unterscheidet, erfasst werden. Bei Verfasserschriften erscheint in jedem Fall der vollständige Verfassername im Indexeintrag, ggf. werden weitere Ordnungselemente ergänzt. Eine besondere Herausforderung stellten die Gruppenschriften nach $\mathrm{Pl}$ dar, die mit verschiedenen Kodierungen eingegeben werden müssen. Auch

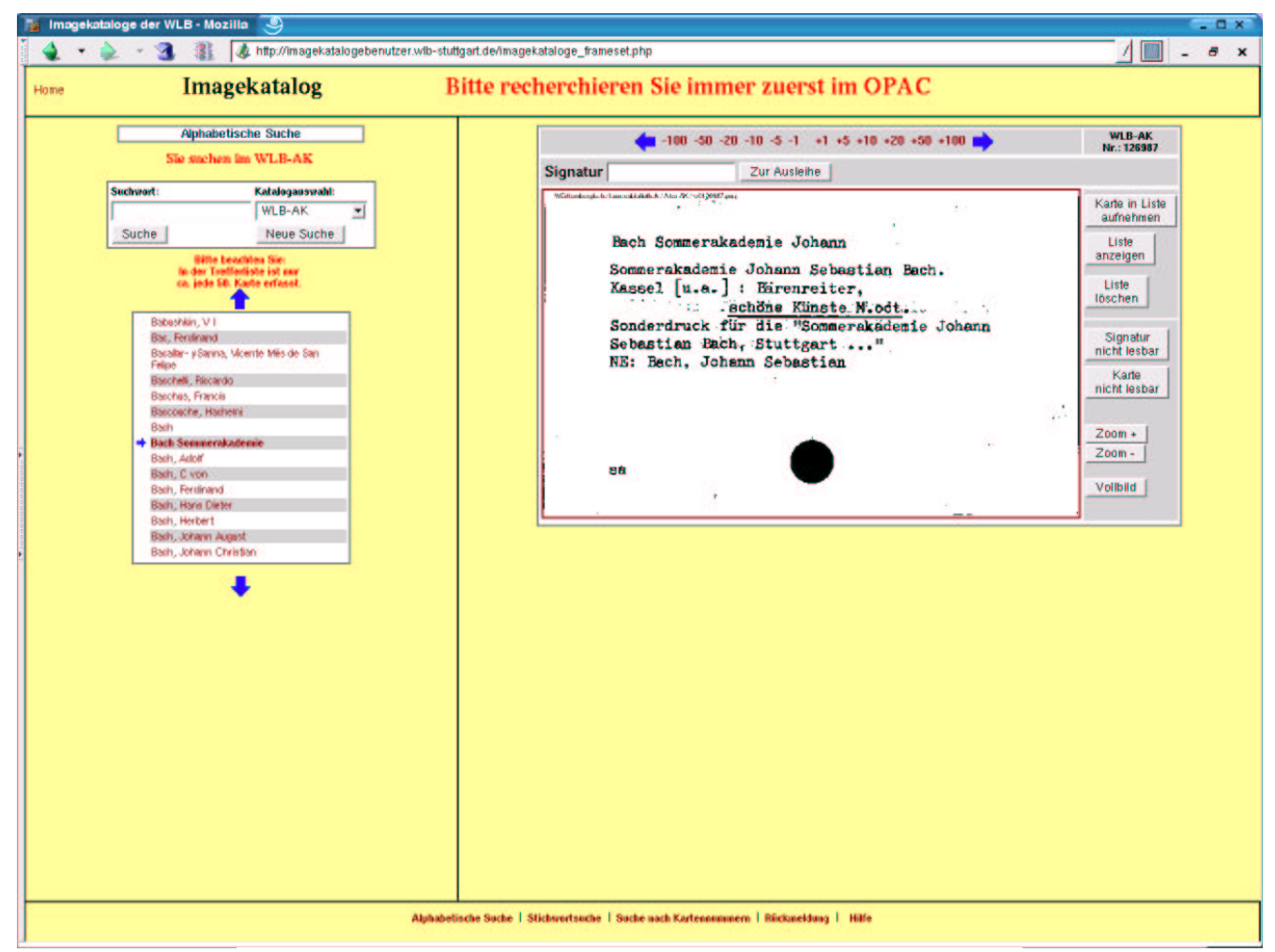

Die Recherchemaske des Imagekataloges (Entwurf) 
die Strukturierung von Doppelnamen, Nachnamen mit Präfixen und Namenszusätzen sowie Persönlichen Namen war keine einfache Aufgabe.

Anfang Juli konnten die Arbeiten der Firma vor Ort beginnen. Im Sitzungszimmer wurden an zwei Hochleistungsscannern in sechs Wochen ca. 3,1 Mio. Karten digitalisiert und einer ersten Qualitätskontrolle unterzogen. Die Arbeiten erfolgten parallel zur Asbestsanierung bei Außentemperaturen von teilweise bis zu 40 Grad. Die Mitarbeiter der Bibliothek hatten in mehreren Führungen Gelegenheit, den ScanBetrieb zu besichtigen. Im August begann Mikro Univers in Berlin mit der Indexierung der Karten. Mitte November war die Lieferung der Images zusammen mit den Indexeinträgen abgeschlossen. Das Projektende hat sich damit nur um zwei Wochen verzögert.

Nach der Auslieferung der ersten konfektionierten Daten wurden die Arbeiten an der Recherchemaske und an der Benutzer-Hilfe intensiviert.

\section{Benutzung des Kataloges}

Der Imagekatalog bietet im wesentlichen eine alphabetische Suche in den elektronischen Katalogkarten des Alphabetischen Hauptkataloges, des Kataloges der BfZ und des Alphabetischen Kataloges der Musikabteilung. Im Menü „Katalogauswahl“ wählt der Benutzer zunächst den gewünschten Katalog an. Der Sucheinstieg erfolgt über einen Index, in dem ca. jede 50. Karte erfasst ist. Dies bedeutet, dass aus dem PI-Kopf jeder 50. Titelkarte bzw. jeder Karte nach einer Leitkarte ein Indexeintrag nach den oben angedeuteten Prinzipien gebildet wurde. Es ist also nur eine Suche nach sachlichen PI-Ordnungswörtern oder nach Verfassernamen möglich, nicht nach anderen Kategorien der Titelaufnahme, wie dies im OPAC der Fall ist.

Nach der Eingabe des Suchwortes klappt der Index an der entsprechenden Stelle im Alphabet auf. Durch Anklicken des gewünschten Eintrages oder des Eintrages, der diesem alphabetisch am nächsten ist, erscheint auf der rechten Seite des Bildschirmes die dem Eintrag zugeordnete Katalogkarte in elektronischer Form. Da nur jede 50. Karte erfasst ist, wird es sich in den meisten Fällen nicht exakt um den gesuchten Titel handeln. Der Benutzer muss dann vorwärts und rückwärts blättern, bis er den gewünschten Treffer gefunden hat. Das Blättern erfolgt auf einer Schaltfläche über dem Kartenimage in Einer-, Fünfer-, Zehner-, Zwanziger-, Fünfziger- und Hunderterschritten. Ist der gesuchte Treffer gefunden, so kann nach der Eingabe der Signatur die Ausleihfunktion angesteuert werden.

Da einige der Titelnachweise im Imagekatalog auch bereits im Verbund katalogisiert oder konvertiert sind, ist der Benutzer angehalten, immer zuerst im OPAC zu suchen und erst dann 
in den Imagekatalog einzusteigen und ggf. über diesen zu bestellen.

Nicht lesbare Signaturen oder Images können der Bibliothek zur Nachbearbeitung gemeldet werden. Die Möglichkeit, eine Trefferliste anzulegen, sowie das Kartenimage zu vergrößern oder zu verkleinern, besteht ebenfalls. Auch eine E-Mail-Funktion ist integriert.

Neben der alphabetischen Suche wird eine Suche nach Kartennummern sowie eine Stichwortsuche angeboten. Bei der Stichwortsuche erfolgt eine Volltextrecherche über die Indexeinträge (nicht über den Volltext der Katalogkarten!). Dies bedeutet, dass auch Suchwörter, die an zweiter oder dritter Stelle im Indexeintrag stehen, recherchierbar sind. Es bedeutet jedoch nicht unbedingt, dass bei der Meldung „Kein Treffer gefunden“ tatsächlich kein Titel vorhanden ist, da über die Suche nur die Indexeinträge jeder 50 . Karte ausgewertet werden. Der Benut- zer muss bei einer solchen Meldung dann zurück in die alphabetische Suche gehen.

Auch wenn der Imagekatalog den traditionellen Ordnungsprinzipien des Alphabetischen Kataloges folgt, stellt seine Einführung dennoch einen Qualitätssprung dar. Der Hauptvorteil für den Nutzer liegt in der ortsunabhängigen Verfügbarkeit des Katalogs im Internet. Damit wird eine nicht unwesentliche Lücke im Online-KatalogAngebot der WLB geschlossen: Mit der Freischaltung des Imagekataloges wird der Gesamtbestand der WLB online recherchierbar sein.

Als nächste Projektstufe steht die OCR-Erfassung aller Images an, die eine „echte“ Volltextsuche über alle Karten des Alphabetischen Kataloges ermöglichen soll und damit den Nutzern deutlich erweiterte Recherchemöglichkeiten bieten wird.

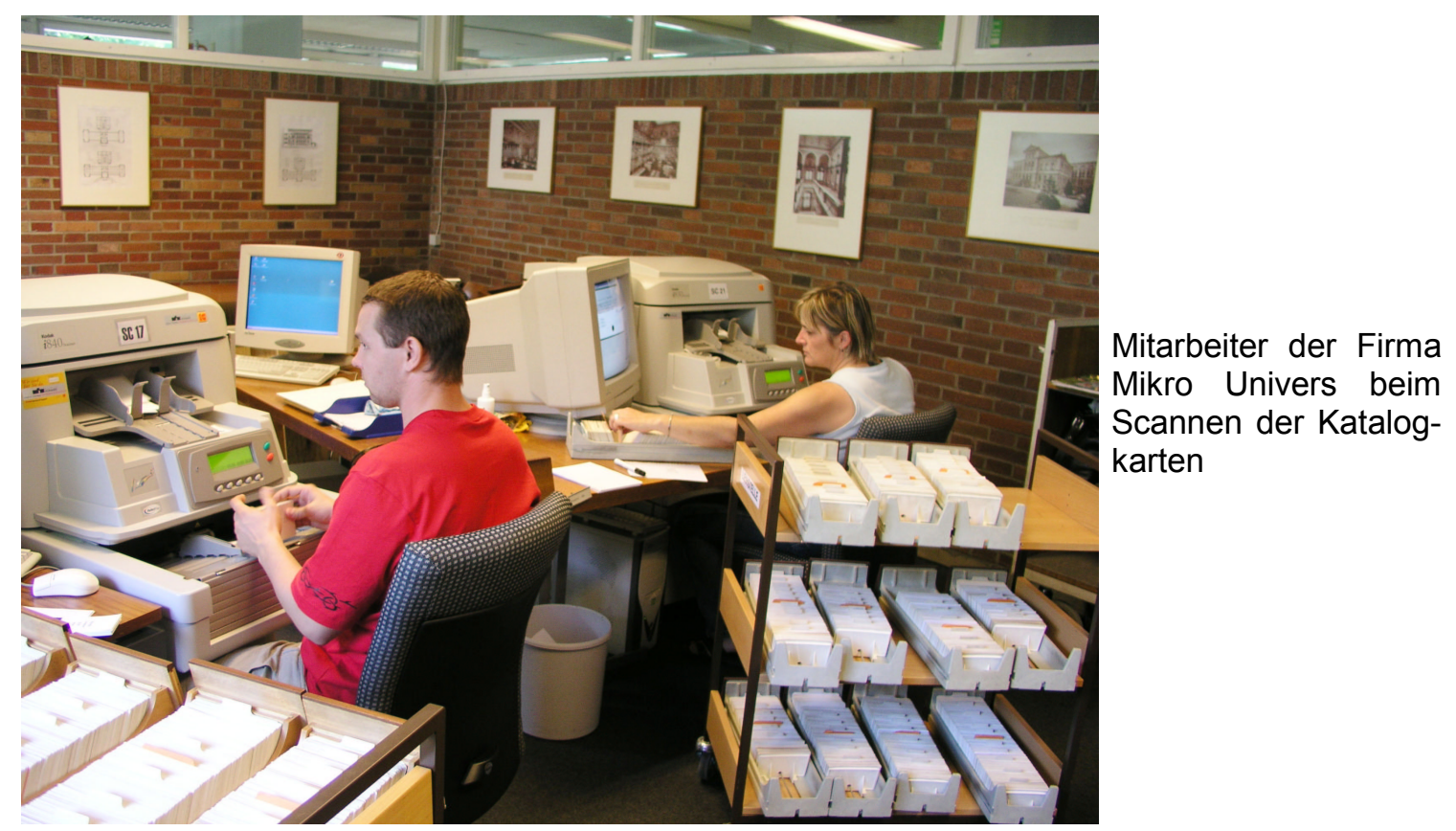

